\title{
FTIR spectroscopy reveals the concentration dependence of cellular modifications induced by anticancer drugs
}

\author{
Régis Gasper ${ }^{\mathrm{a}, *}$, Tatjana Mijatovic ${ }^{\mathrm{b}}$, Robert Kiss ${ }^{\mathrm{b}}$ and Erik Goormaghtigh ${ }^{\mathrm{a}}$ \\ ${ }^{\mathrm{a}}$ Laboratory for the Structure and Function of Biological Membranes, Center for Structural Biology \\ and Bioinformatics, Université Libre de Bruxelles, Brussels, Belgium \\ ${ }^{\mathrm{b}}$ Laboratory of Toxicology, Institute of Pharmacy, Université Libre de Bruxelles, Brussels, Belgium
}

\begin{abstract}
Large-scale screening to determine the mechanisms of anti-cancer actions of chemical libraries still presents technical challenges that are beyond the capabilities of conventional methods used in cellular or molecular biology. We recently demonstrated in a proof-of-concept study that infrared (IR) spectrum of cells exposed to anticancer drugs could be used to classify their mechanisms of actions. This study highlighted the fact that molecules inducing unique metabolic modifications could be selected for further pharmacological improvements. We show in this paper that drug concentration is an important parameter to be taken into account when analyzing mechanisms of anti-cancer actions by means of FTIR. The data indeed demonstrated that distinct spectral modifications occur in human PC-3 prostate cancer cells when exposed to ouabain at $10 \times \mathrm{IC}_{50}$ versus $1 \times \mathrm{IC}_{50}$. Longer incubation times at $1 \times \mathrm{IC}_{50}$ never resulted in spectral modifications fitting with those observed at $10 \times \mathrm{IC}_{50}$. Keywords: IR spectroscopy, cancer, drug
\end{abstract}

\author{
Abbreviations \\ IR: Infrared \\ FTIR: Fourier Transform Infrared \\ h: Hours
}

\section{Introduction}

Infrared spectroscopy has become a powerful tool for the study of biological systems. This technique records a fingerprint of all the molecules present in the cells: lipids, sugars, proteins, nucleic acids, nucleotides, vitamins, etc. It senses not only the chemical nature of the cell molecules but also their conformations. It is for instance very sensitive to protein conformation [6,13]. This approach has already been used in several fields such as classification of bacterial sub-species [12,15] or differentiation of tumor and normal cells $[1,2,16]$. In our previous study, we have shown that IR spectroscopy is sensitive

\footnotetext{
* Corresponding author: Régis Gasper, Center for Structural Biology and Bioinformatics, Laboratory for the Structure and Function of Biological Membranes, Campus Plaine CP206/02, Université Libre de Bruxelles, Bld du Triomphe 2, CP206/2, B1050 Brussels, Belgium. Tel.: +32 265053 62; Fax: +32 265053 82; E-mail: regis.gasper@ulb.ac.be.
} 
enough to characterize the metabolic changes induced by ouabain, a compound belonging to the cardiotonic steroid family, as a function of the exposure time to the molecule [4]. Draux et al. also report that subtoxic doses of gemcitabine, an anti-tumor drug, can be monitored [3]. Cardiotonic steroids include two major chemical classes, i.e. bufadienolides and cardenolides, which are well-known inotropic drugs for the treatment of congestive heart failure; several studies recently underlined their anti-tumoral actions (reviewed in $[9,10]$ ). The specific binding of these molecules to the $\mathrm{Na}^{+} / \mathrm{K}^{+}$-ATPase pump can activate various cell death pathways in some tumour cells [10]. Studies on several cardenolides and cell lines revealed that the cytotoxic concentration of these molecules can vary by two orders of magnitude while the rate of the $\mathrm{Na}^{+} / \mathrm{K}^{+}$-ATPase inhibition remains nearly the same [17]. The aim of this study is to investigate whether the concentration of ouabain used to treat human PC-3 prostate cancer cells may determine different mode of action revealed by FTIR spectroscopy.

\section{Materials and methods}

\subsection{Cell culture and treatment}

The human prostate cancer PC-3 (CRL-1435) cell line was obtained from the American Type Culture Collection (ATCC, Manassas, VA, USA) and was maintained according to the supplier's instructions. The cells were incubated at $37^{\circ} \mathrm{C}$ in sealed (airtight) Falcon plastic dishes (Nunc, Invitrogen SA, Merelbeke, Belgium) in a humidified atmosphere of $5 \% \mathrm{CO}_{2}$. The cells were kept in exponential growth in RPMI medium supplemented with $10 \%$ fetal bovine serum (FBS), $1 \%$ penicillin/streptomycin (an antibiotic/antimycotic solution) and 1\% kanamycin to prevent mycoplasms. Cell culture medium and FBS were purchased from Gibco (Invitrogen, Merelbeke, Belgium). Penicillin/strepromycin and kanamycin solutions were provided by Sigma-Aldrich SA (Bornem, Belgium). Ouabain was purchased from Acros Organics (Geel, Belgium).

For FTIR spectroscopy, cells were detached by means of a five-minute treatment with trypsin/EDTA buffer (Gibco, Invitrogen SA, Merelbeke, Belgium). The reaction was stopped by adding $1 \mathrm{ml}$ of culture medium. The cells were pelleted by a 2-minute centrifugation $(300 \mathrm{~g})$, and washed three times in isotonic solution $(\mathrm{NaCl}, 0.9 \%)$ to ensure complete removal of trypsin and culture medium. They were then suspended in $30 \mu \mathrm{l}$ of the $\mathrm{NaCl}$ solution.

\subsection{FTIR spectroscopy}

All measurements were carried out on a Bruker Equinox 55 FTIR spectrometer (Bruker, Karlsruhe, Germany) equipped with a liquid $\mathrm{N}_{2}$-refrigerated mercury cadmium Telluride detector. All spectra were recorded by attenuated total reflection (for a review, see [5]). A diamond internal reflection element was used on a Golden Gate Micro-ATR from Specac (Orpington, UK). The angle of incidence was 45 degrees. $0.5 \mu \mathrm{l}$ of cell pellet re-suspended in about twice the volume of the initial pellet was deposited on the diamond crystal (about $3 \times 10^{4}$ cells per smear). The sample was quickly evaporated in $\mathrm{N}_{2}$ flux to obtain a homogenous film of whole cells, as ascertained by microscope examination. The FTIR measurements were recorded between 4000 and $800 \mathrm{~cm}^{-1}$. Each spectrum was obtained by averaging 256 scans recorded at a resolution of $2 \mathrm{~cm}^{-1}$. For statistical validation the cells were grown in three independent boxes for each experiment and three samplings were taken from each box, thus generating a total of 9 spectra per condition. For each case, 3 boxes of non treated cells were grown, collected and analyzed in the same condition as the treated ones as a control, generating a total of 27 non-treated cells 
spectra. These spectra show no statistically significant difference from one culture to another according to a non-parametric Wilcoxon test at a significance level of $0.5 \%$ (not shown).

\subsection{Data analysis}

The FTIR data were preprocessed as follows. First, the water vapor contribution was subtracted, and then the spectra were baseline-corrected and normalized for equal area between 1765 and $950 \mathrm{~cm}^{-1}$. The spectra were finally smoothed at a final resolution of $4 \mathrm{~cm}^{-1}$ by apodization of their Fourier transform by a Gaussian line. The processing of the spectra and Wilcoxon tests were carried out by means of specific software generated in our lab running under Matlab (Mathworks Inc.).

\section{Results and discussion}

To evaluate the influence of ouabain concentration on metabolic modifications induced in PC-3 cells, we used it at two concentrations: the $\mathrm{IC}_{50}(36 \mathrm{nM})$ and 10 times higher $\left(10 \times \mathrm{IC}_{50}\right)$. The $\mathrm{IC}_{50}$ was evaluated by means of a colorimetric MTT assay as previously reported [4]. The incubation times were 0,6 and $24 \mathrm{~h}$. Longer exposure times at $10 \times \mathrm{IC}_{50}$ revealed to be highly cytotoxic. Each concentration or exposure time is represented by nine spectra and 27 spectra of non-treated cells from 9 independent samples were also recorded (see Section 2).

Figure 1 reports FTIR difference spectra between pairs of conditions. The two spectra in (A) are the difference between the mean spectrum of PC-3 cells exposed for $6 \mathrm{~h}$ to ouabain and non-treated cells.

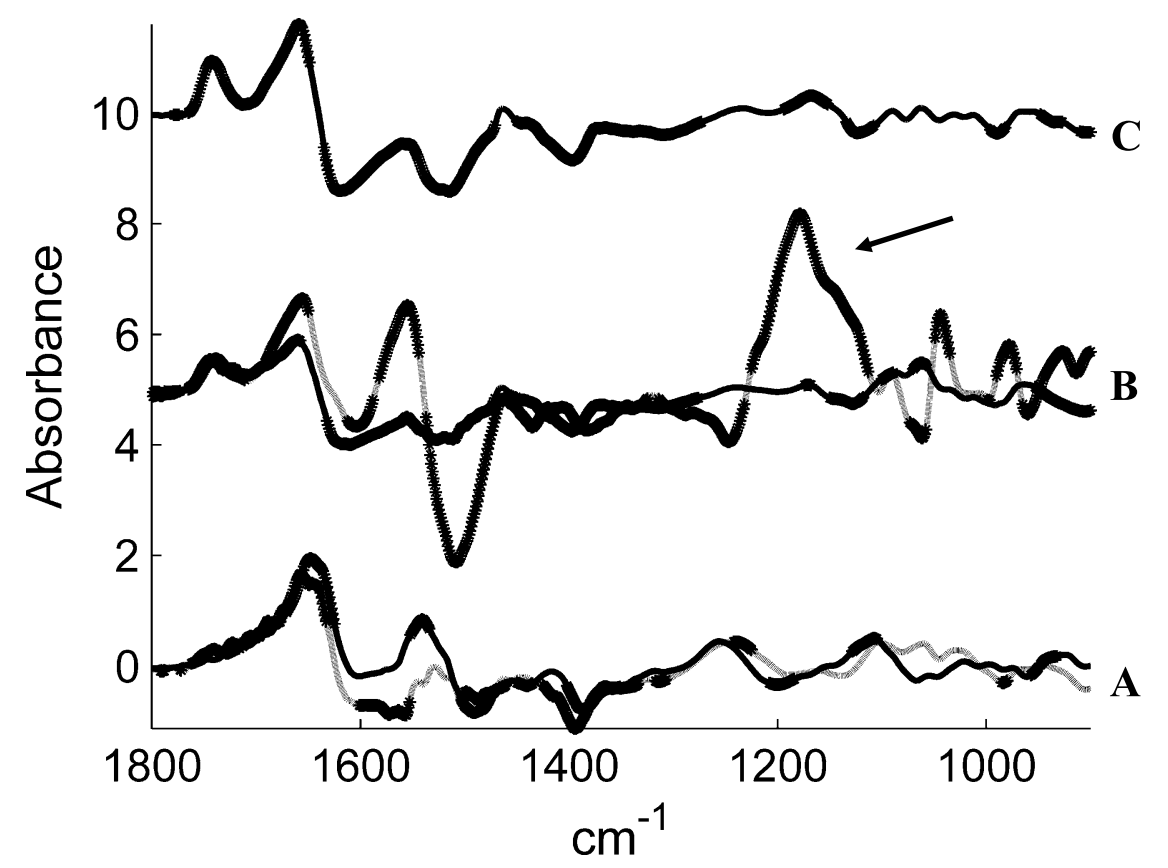

Fig. 1. Difference between the mean spectra of PC-3 cells recorded in two experimental conditions: (A) $6 \mathrm{~h}$ non-treated cells, (B) 24-6 h, (C) 48-6 h. A Wilcoxon test was computed at every wavenumber with a significance level of $\alpha=0.5 \%$. Each marked wavenumber (thick line) represents a statistically significant difference between the means. Results obtained at the $\mathrm{IC}_{50}$ are presented by black lines while $10 \times \mathrm{IC}_{50}$ results appear in grey. 
After $6 \mathrm{~h}$ of incubation, the effects of both drug concentrations were highly similar, as suggested by the similar shape of the difference spectra. Yet, the lipids and amide I area $\left(1760-1500 \mathrm{~cm}^{-1}\right)$ seems to be weakly affected by ouabain at its $\mathrm{IC}_{50}$ concentration while at a $10 \times \mathrm{IC}_{50}$ the whole spectral range is characterized by marked modifications.

The spectra in (B) are the differences between the mean spectra recorded after 24 and $6 \mathrm{~h}$ of exposure to ouabain. Between 6 and $24 \mathrm{~h}$ the differences appear highly distinct for both concentrations. Yet, between 1760 and $1700 \mathrm{~cm}^{-1}$ (lipids), spectral changes appear to be similar in shape and intensity. The Amide I region, 1700-1600 $\mathrm{cm}^{-1}$, displays slight differences in the intensity of difference spectra but, overall, the shapes are identical and wavenumbers that statistically differ between 24 and $6 \mathrm{~h}$ incubation times are not notably different. This region is known to be characteristic of protein secondary structure [5].

One of the most impressive dissimilarity was the large positive peak appearing between 1230 and $1100 \mathrm{~cm}^{-1}$ in the $10 \times \mathrm{IC}_{50}$ difference spectrum. Absorption in this spectral region is mainly dominated by contribution from phosphates or carbohydrates $[8,11]$. The second major distinction is found in the $1600-1470 \mathrm{~cm}^{-1}$ area, largely related to proteins [11] and displayed the characteristic shape of a peak shift, which can be observed on the original spectrum (from 1536 to $1542 \mathrm{~cm}^{-1}$, not shown). The third region, $1100-900 \mathrm{~cm}^{-1}$, looks completely different at both concentrations.

We also attempted to investigate whether longer incubation times at the $\mathrm{IC}_{50}$ leads to the same metabolic perturbations as $24 \mathrm{~h}$ of exposure to $10 \times \mathrm{IC}_{50}$. (C) represents the difference spectrum between 48 and $6 \mathrm{~h}$ with ouabain treatment at $\mathrm{IC}_{50}$. Difference in spectra arising from $48-6 \mathrm{~h}$ or $24-6 \mathrm{~h}$ are almost identical, yet with some minor divergences in the intensity (essentially in the lipid-protein spectral absorption zone, roughly $1800-1400 \mathrm{~cm}^{-1}$ ) strongly suggesting that concentration but not exposure time is responsible for the induction of another type of metabolic perturbation in PC-3 cells.

Infrared spectroscopy was used to evaluate the impact of ouabain on prostate PC-3 cancer cells. High ouabain concentration $\left(10 \times \mathrm{IC}_{50}\right)$ induces changes in the chemical composition of the cells that never appear at lower concentration $\left(1 \times \mathrm{IC}_{50}\right)$ even when longer exposure times are considered. Overall, our data suggest that a completely distinct mechanism of action has been triggered at higher concentration. The importance of drug concentration for the mechanism of anti-tumor action has been reported in number of studies and nicely demonstrated by Rebbaa et al. [14] showing that doxorubicin induced at least three distinct types of cell death, senescence, apoptosis and a type of necrosis, which were concentration dependent $(0.1,1$ and $10 \mu \mathrm{M}$, respectively). Concentration is potentially even more important when working with cardiotonic steroids. Indeed, the sodium pump mediates CS-induced effects in a compound, concentration and cell type-specific manner (for review see [9]). Interestingly, Huang et al. [7] have demonstrated that increasing ouabain concentrations induced different effects in PC-3 prostate cancer cells. Low concentrations of ouabain induced an increase in Par-4 expression which sensitized the cells to cytotoxicity, while high concentrations induced a loss of deltapsim, sustained reactive oxygen species (ROS) production and apoptosis.

In conclusion, the present study further supports the importance of drug concentration selection and suggests that FTIR spectroscopy could become a new method of choice for its evaluation.

\section{Acknowledgements}

This research has been supported by a grant from Interuniversity Attraction Poles (IAP) P6/19 (Belgium) and the National Fund for Scientific Research (FRFC 2.4533.10 and 2.4588.06). Régis Gasper 
is the holder of a grant from the "IAP", Université Libre de Bruxelles, Brussels, Belgium. Erik Goormaghtigh and Robert Kiss are Directors of Research with the "National Fund for Scientific Research", Belgium.

\section{References}

[1] L. Chiriboga, P. Xie, V. Vigorita, D. Zarou, D. Zakim and M. Diem, Infrared spectroscopy of human tissue. II. A comparative study of spectra of biopsies of cervical squamous epithelium and of exfoliated cervical cells, Biospectrosc. 4 (1998), $55-59$.

[2] L. Chiriboga, P. Xie, H. Yee, V. Vigorita, D. Zarou, D. Zakim and M. Diem, Infrared spectroscopy of human tissue. I. Differentiation and maturation of epithelial cells in the human cervix, Biospectrosc. 4 (1998), 47-53.

[3] F. Draux, P. Jeannesson, C. Gobinet, J. Sule-Suso, J. Pijanka, C. Sandt, P. Dumas, M. Manfait and G.D. Sockalingum, IR spectroscopy reveals effect of non-cytotoxic doses of anti-tumour drug on cancer cells, Anal. Bioanal. Chem. 395 (2009), 2293-2301.

[4] R. Gasper, J. Dewelle, R. Kiss, T. Mijatovic and E. Goormaghtigh, IR spectroscopy as a new tool for evidencing antitumor drug signatures, Biochim. Biophys. Acta 1788 (2009), 1263-1270.

[5] E. Goormaghtigh, V. Raussens and J.M. Ruysschaert, Attenuated total reflection infrared spectroscopy of proteins and lipids in biological membranes, Biochim. Biophys. Acta 1422 (1999), 105-185.

[6] E. Goormaghtigh, J.M. Ruysschaert and V. Raussens, Evaluation of the information content in infrared spectra for protein secondary structure determination, Biophys. J. 90 (2006), 2946-2957.

[7] Y.T. Huang, S.C. Chueh, C.M. Teng and J.H. Guh, Investigation of ouabain-induced anticancer effect in human androgenindependent prostate cancer PC-3 cells, Biochem. Pharmacol. 67 (2004), 727-733.

[8] P. Lasch, M. Boese, A. Pacifico and M. Diem, FT-IR spectroscopic investigations of single cells on the subcellular level, Vib. Spectrosc. 28 (2002), 147-157.

[9] T. Mijatovic, L. Ingrassia, V. Facchini and R. Kiss, $\mathrm{Na}^{+} / \mathrm{K}^{+}$-ATPase alpha subunits as new targets in anticancer therapy, Expert Opin. Ther. Targets 12 (2008), 1403-1417.

[10] T. Mijatovic, E. van Quaquebeke, B. Delest, O. Debeir, F. Darro and R. Kiss, Cardiotonic steroids on the road to anticancer therapy, Biochim. Biophys. Acta 1776 (2007), 32-57.

[11] D. Naumann, Infrared spectroscopy in microbiology, in: Encyclopedia of Analitical Chemistry, R.A. Meyers, ed., Wiley, Chichester, UK, 2000, pp. 102-131.

[12] D. Naumann, D. Helm and H. Labischinski, Microbiological characterizations by FT-IR spectroscopy, Nature 351 (1991), $81-82$.

[13] K.A. Oberg, J.M. Ruysschaert and E. Goormaghtigh, The optimization of protein secondary structure determination with infrared and circular dichroism spectra, Eur. J. Biochem. 271 (2004), 2937-2948.

[14] A. Rebbaa, X. Zheng, P.M. Chou and B.L. Mirkin, Caspase inhibition switches doxorubicin-induced apoptosis to senescence, Oncogene 22 (2003), 2805-2811.

[15] C.A. Rebuffo, J. Schmitt, M. Wenning, F. von Stetten and S. Scherer, Reliable and rapid identification of Listeria monocytogenes and Listeria species by artificial neural network-based Fourier transform infrared spectroscopy, Appl. Environ. Microbiol. 72 (2006), 994-1000.

[16] B. Rigas and P.T.T. Wong, Human colon adenocarcinoma cell-lines display infrared spectroscopic features of malignant colon tissues, Cancer Res. 52 (1992), 84-88.

[17] E. van Quaquebeke, G. Simon, A. Andre, J. Dewelle, M. El Yazidi, F. Bruyneel, J. Tuti, O. Nacoulma, P. Guissou, C. Decaestecker, J.C. Braekman, R. Kiss and F. Darro, Identification of a novel cardenolide ( $2^{\prime \prime}$-oxovoruscharin) from Calotropis procera and the hemisynthesis of novel derivatives displaying potent in vitro antitumor activities and high in vivo tolerance: structure-activity relationship analyses, J. Med. Chem. 48 (2005), 849-856. 


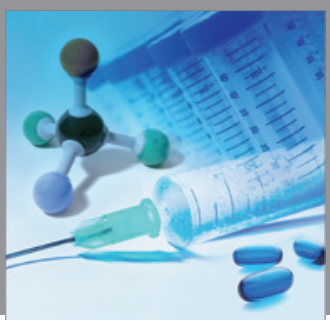

International Journal of

Medicinal Chemistry

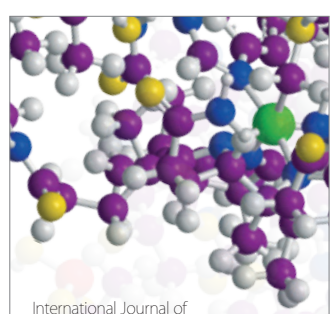

Carbohydrate Chemistry

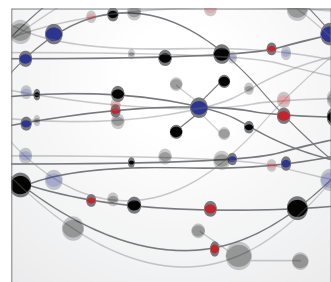

The Scientific World Journal
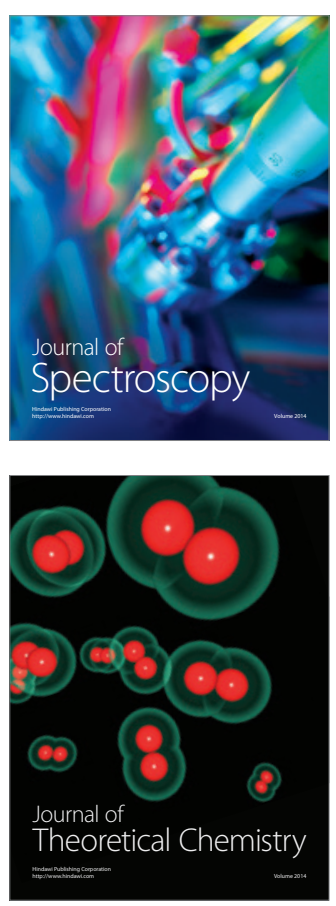
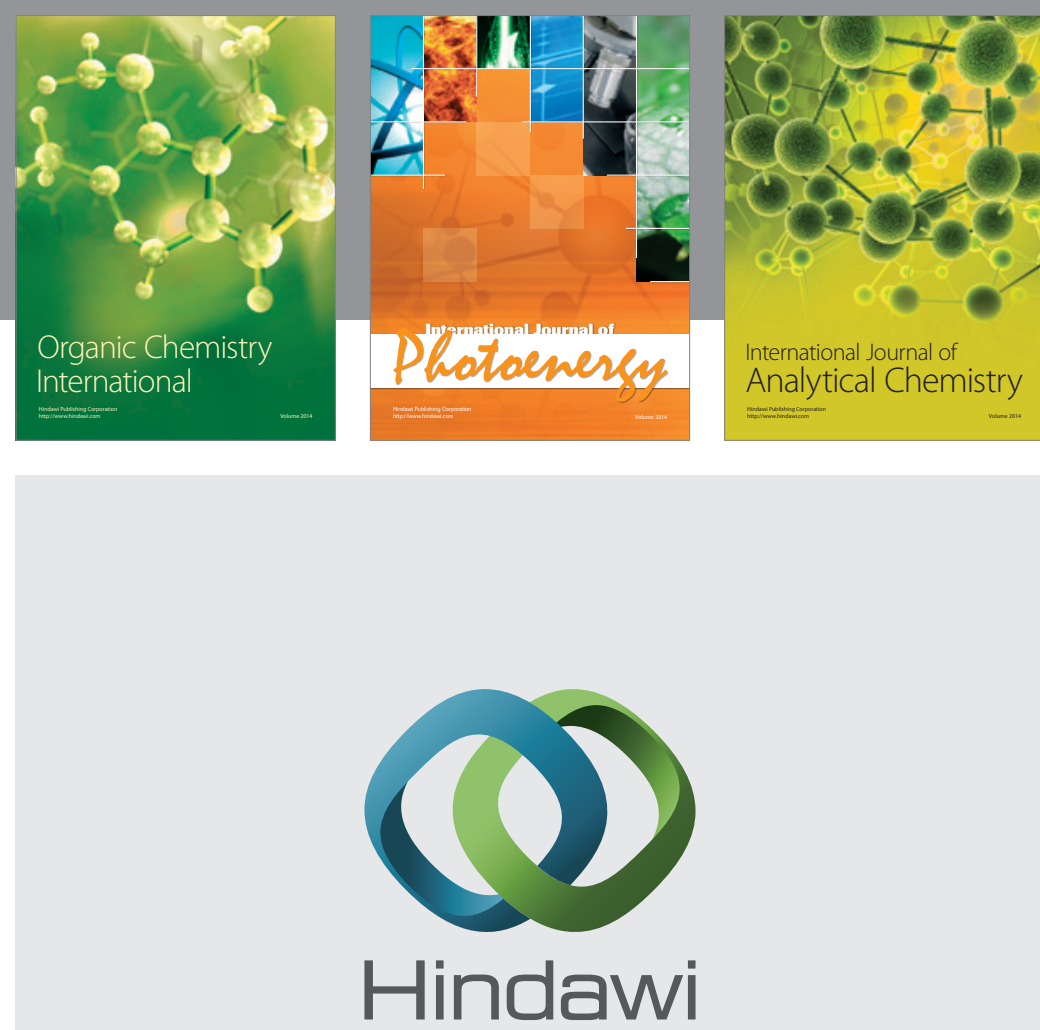

Submit your manuscripts at

http://www.hindawi.com
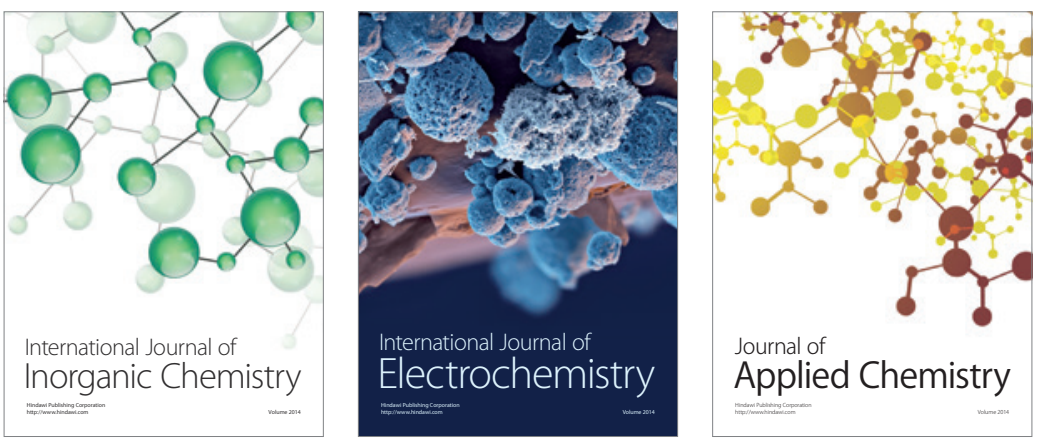

Journal of

Applied Chemistry
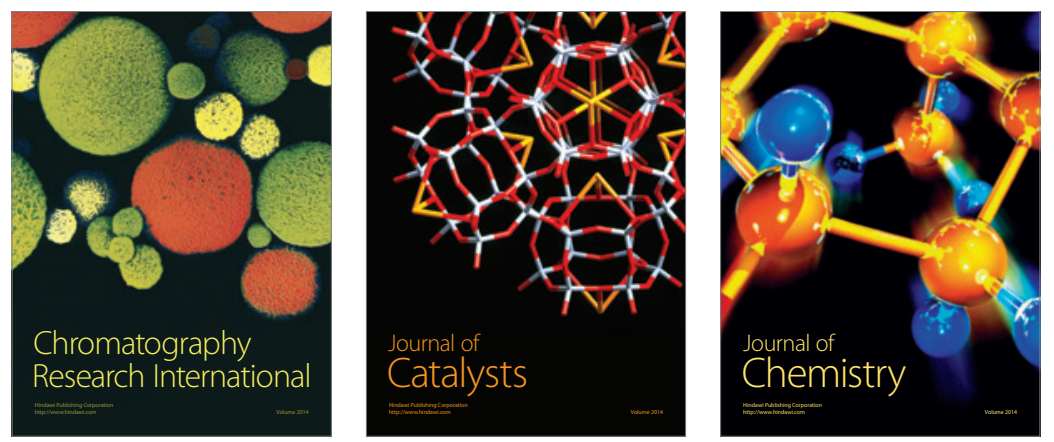
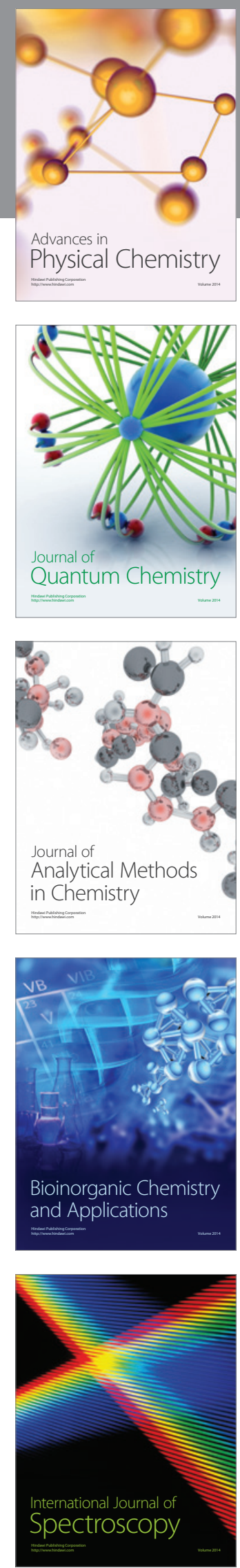